\title{
The Generation of Minimal Triangle Graphs
}

\section{By Robert Bowen}

Let $T$ be a finite set of triples (sets of three distinct elements). We define $G(T)$ to be the 1 -skeleton of the 2 -complex formed by $T$ (i.e. the graph with elements of triples of $T$ as vertices and two vertices adjacent iff they are distinct and lie in a common member of $T$ ). $T$ is a minimal triangle set (MTS) of order $n$ if (i) $G(T)$ has $n$ vertices and is connected, and (ii) $G(T-\{t\})$ satisfies (i) for no $t$ in $T$. In such a case $G(T)$ is a minimal triangle graph (MTG) of order $n$. Our problem is to generate all (nonisomorphic) MTS's of a given order or equivalently (by Theorem 1) all MTG's of that order.

Theorem 1. If $G$ is an $M T G$, then $G=G(T)$ for a unique MTS T.

Proof. Suppose $T$ and $T^{\prime}$ are MTS's with $G=G(T)=G\left(T^{\prime}\right)$ and $t \in T, t \notin T^{\prime}$. Let $t=\left\{P_{1}, P_{2}, P_{3}\right\}$. As $t \notin T^{\prime}$ and $G=G\left(T^{\prime}\right)$, the pairs $\left\{P_{1}, P_{2}\right\},\left\{P_{1}, P_{3}\right\}$, and $\left\{P_{2}, P_{3}\right\}$ are in some triples $\left\{P_{1}, P_{2}, P_{4}\right\},\left\{P_{1}, P_{3}, P_{5}\right\}$, and $\left\{P_{2}, P_{3}, P_{6}\right\}$ of $T^{\prime}$. Then all of $P_{1} P_{4}, P_{4} P_{2}, P_{2} P_{6}, P_{6} P_{3}, P_{3} P_{5}$, and $P_{5} P_{1}$ are all edges of $G$ not lying on triangle $t$. Hence $P_{1} P_{4} P_{2} P_{6} P_{3} P_{5} P_{1}$ is a cycle in $G(T-\{t\})$ and $G(T-\{t\})$ is connected and has the same vertices as $G(T)$, contradicting $T$ an MTS.

If $P$ is a vertex of a graph $G$, we let $G_{P}$ denote the graph formed by removing $P$ and incident edges from $G$.

LEMMA 1. If $G$ is a connected graph, then $G_{P}$ is connected for some vertex $P$ of $G$.

Proof. A spanning tree of $G$ is a tree subgraph of $G$ containing all the vertices of $G$. As $G$ is connected, from [2] we know that $G$ has a spanning tree $K$. Let $P$ be a vertex of $K$ of valency one. Then $K_{P}$ is a spanning tree for $G_{P}$; hence $G_{P}$ is connected.

Lemma 2. If $G(T)$ is connected, then $G(T-\{t\})$ is connected for some $t$ in $T$.

Proof. Form the graph $H(T)$ by taking members of $T$ as vertices and letting a pair of them be adjacent iff they are distinct and have an element in common. Then $H(T)$ is connected iff $G(T)$ is, for there is a path between two vertices in $G(T)$ iff there is one in $H(T)$ between elements of $T$ containing the vertices. As $G(T)$ is connected, so is $H(T)$; and, by Lemma 1 , so is $H(T)_{t}=H(T-\{t\})$ for some $t$ in $T$. Then $G(T-\{t\})$ is connected also.

We note that the same proof applies to any complex; in any connected complex $T$ there is some simplex $t$ such that the 1-skeleton of $T-\{t\}$ is connected and hence $T-\{t\}$ is connected also. An alternate proof of Lemma 2 appears in [1].

THEOREm 2. Let $T$ be an MTS of order $n \geqq 4$. Then there is an MTS $T^{\prime}$ of order $n-1$ or $n-2$ such that $T^{\prime}=T-\{t\}$ for some $t \in T$.

Proof. Let $t \in T$ as in Lemma 2 and set $T^{\prime}=T-\{t\}$. As $T$ is an MTS and $G\left(T^{\prime}\right)$ is connected, $G\left(T^{\prime}\right)$ must have fewer vertices than $G(T)$. As $G(T)$ is connected and $T^{\prime} \neq \varnothing, G\left(T^{\prime}\right)$ cannot have three fewer vertices than $G(T)$. Hence $G\left(T^{\prime}\right)$ has $n-1$ or $n-2$ vertices. Finally suppose $t^{\prime} \in T^{\prime}, T^{\prime \prime}=T^{\prime}-\left\{t^{\prime}\right\}$, with $G\left(T^{\prime \prime}\right)$ connected and having the same vertices as $G\left(T^{\prime}\right)$. Then $G\left(T^{\prime \prime} \cup\{t\}\right)=$ $G\left(T-\left\{t^{\prime}\right\}\right)$ has the same vertices as $G(T)$ and hence is not connected since $T$ is an MTS. Since $G\left(T^{\prime \prime}\right)$ is connected, this means that $t$ has no vertex in common with

Received September 7, 1965. 
$G\left(T^{\prime \prime}\right)$. But $G\left(T^{\prime \prime}\right)$ has the same vertices as $G\left(T^{\prime \prime}\right)$; hence three vertices of $G(T)$ are not in $G\left(T^{\prime}\right)$, a contradiction

Now suppose $n \geqq 4$ and we have all MTS's of order $n-1$ and $n-2$. If we add a triple to those of order $n-2$ with exactly two new vertices in all possible ways and add a triple with exactly one new vertex to those of order $n-1$ in all possible ways, then by Theorem 2 we shall obtain all MTS's of order $n$. We are left with the problem of sorting out isomorphic MTS's. For this purpose a routine was developed for testing two graphs for isomorphism (described in the next paragraph). As we generate an MTS $T$ we compare $G(T)$ against the graphs of the MTS's already generated. If $G(T)$ is isomorphic to $G\left(T^{*}\right)$ with $T^{*}$ already generated, then Theorem 1 assures us that $T$ and $T^{*}$ are isomorphic. Hence $T$ is discarded in such a case. If no such $T^{*}$ has been generated, then $T$ is new, $T$ is added to the list of MTS's of order $n$, and $G(T)$ is retained for future testing.

The isomorphism routine handles connected graphs with 35 or fewer vertices. For $P, Q$ vertices of a graph $G$ let $a(P, Q)=1$ if $P$ and $Q$ are adjacent and 0 otherwise. Set $b_{0}(P)=1$ and $b_{k+1}(P)=\sum_{Q} a(P, Q) b_{k}(Q)$ for all $P$. Note that $b_{1}(P)$ is just the valence of $P$. According to the numbers $b_{1}, b_{2}$ and $b_{3}$ the vertex sets of each of the two graphs being tested are divided into classes and the classes of the two graphs are matched if possible. If a vertex is the only member of its class, it is matched with the corresponding vertex of the other graph. With a partial matching of vertices a few simple tests search for a local contradiction or a "forced" local extension. During these tests a desirable vertex is picked out for arbitrary matching in case this is necessary. After this vertex is matched, the process is repeated. We move up and down our "possibility tree" in the usual back-track method until a final contradiction or an isomorphism is attained.

Computations were carried out on an IBM 7094. In the table below $M(n)$ is the number of MTS's (or MTG's) of order $n$. We started from the single MTS of order 3 , the triangle. We generated those of order thirteen and less in approximately twenty minutes. In a test of the ismorphism routine, in four minutes isomorphisms were constructed between 9600 pairs of isomorphic triangular graphs with 12 vertices. $M(n)$ was checked by hand for $n \geqq 7$.

$\begin{array}{rrrr}n & M(n) & n & M(n) \\ 4 & 1 & 9 & 48 \\ 5 & 2 & 10 & 117 \\ 6 & 4 & 11 & 307 \\ 7 & 9 & 12 & 821 \\ 8 & 19 & 13 & 2277\end{array}$

Order 4:

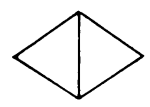

Order 5:
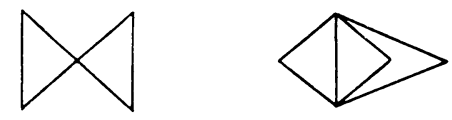

Order 6:
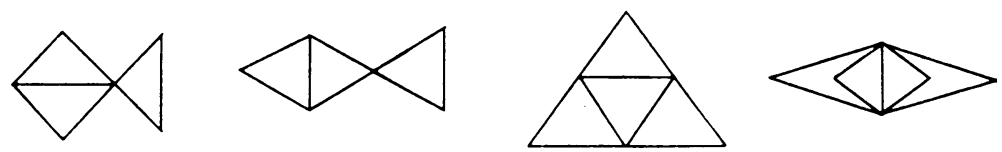
The author is indebted to the University of California (Berkeley) Computer ('enter for its cooperation.

University of California

Berkeley, California

1. Richard Chandler \& Robert Spira, Enumeration of 2-complexes. (To appear.)

2. D. KöNIG, Theorie der endlichen und unendlichen Graphen, Chelsea, New York, 1950, p. 52. MR 12, 195 .

\section{Generation of Triangulations of the Sphere}

\section{By Robert Bowen and Stephen Fisk}

It is easily seen that there is only one triangulation of the sphere with four vertices and one with five. This paper concerns an algorithm for finding all (nonisomorphic) triangulations of the 2 -sphere with $N$ vertices from those with $N-1$. "Triangulation" shall always refer to a triangulation of the 2 -sphere. First we develop a method for generating all triangulations with $N$ vertices which may yield several triangulations of the same isomorphism type, and then we describe an isomorphism routine for eliminating these duplications.

Let $T$ be a triangulation with $N \geqq 5$ vertices, $E$ edges, and $F$ faces. Let $X_{k}$ denote the number of vertices of $T$ of valency $k$. Then $3 F=2 E$ as each face is a triangle and each edge is on two faces, and $2 E=\sum k X_{k}$ as each edge is incident to two vertices. Hence $6 F-6 E=-2 E=-\sum k X_{k}$ and by Euler's formula we have

$$
12=6 N+6 F-6 E=6 N-\sum k X_{k}=\sum X_{k}(6-k) .
$$

Since $\sum X_{k}(6-k)$ is positive, $T$ must have a vertex of valency less than six. Because every edge of $T$ must lie on two distinct triangular faces, each vertex must have valency greater than two. Letting $Q$ be a vertex of minimal valency, $Q$ must have valency three, four, or five.

Case 1. Suppose $Q$ has valency three. Then, about $Q, T$ has the form shown in Fig. 1. Removing $Q$ and the edges $Q P_{k}$, we obtain a triangulation $T^{\prime}$ with $N-1$ vertices. Thus we obtain $T$ if we add the point $Q$ to the center of the face $P_{1} P_{2} P_{3}$ and add the edges $Q P_{k}(k=1,2,3)$.

Case 2. Suppose $Q$ has valency four. Then, about $Q, T$ has the form shown in Fig. 2. By the Jordan curve theorem either $P_{1}$ is not adjacent to $P_{3}$ or $P_{2}$ is not adjacent to $P_{4}$; say $P_{1}$ is not adjacent to $P_{3}$. Then, removing $Q$ and edges $Q P_{k}$ $(1 \leqq k \leqq 4)$ and adding edge $P_{1} P_{3}$ inside the quadrilateral $P_{1} P_{2} P_{3} P_{4}$, we obtain a triangulation $T^{\prime}$ with $N-1$ vertices. The slight complication here is needed to insure that $T^{\prime}$ is a triangulation; for if $P_{1}$ were adjacent to $P_{3}$ in $T$, then $T^{\prime}$ would have multiple edges and would not be a triangulation. We now obtain $T$ from $T^{\prime}$ by reversing the process.

Case 3. Assume $Q$ has valency five. We claim some $P_{k}$ is adjacent to no $P_{i}$ other than the two shown (Fig. 3 ). Otherwise $P_{1}$ would be adjacent to $P_{3}$ or $P_{4}$, say $P_{3}$. Then by the Jordan curve Theorem, $P_{2}$ could be adjacent to neither $P_{4}$ nor

Received October 25, 1965. 